\title{
Successful Delayed Aortic Surgery for a Patient with Ischemic Stroke Secondary to Aortic Dissection
}

\author{
Ryuta Morihara ${ }^{1}$, Toru Yamashita ${ }^{1}$, Kentaro Deguchi ${ }^{1}$, Keiichiro Tsunoda ${ }^{1}$, \\ Yasuhiro Manabe ${ }^{2}$, Yoshiaki Takahashi ${ }^{2}$, Taijun Yunoki ${ }^{1}$, Kota Sato ${ }^{1}$, Yumiko Nakano ${ }^{1}$, \\ Syoichiro Kono ${ }^{2}$, Yasuyuki Ohta ${ }^{1}$, Nozomi Hishikawa ${ }^{1}$ and Koji Abe ${ }^{1}$
}

\begin{abstract}
:
The diagnosis of aortic dissection (AD) is sometimes difficult within the limited time window of recombinant tissue plasminogen activator (tPA) for ischemic stroke (IS). A 60-year-old man developed sudden left hemiparesis due to IS. During tPA infusion, his blood pressure dropped and consciousness declined. After transfer to our hospital, carotid duplex ultrasonography led to a diagnosis of AD. Emergency surgery was postponed because of the risk of hemorrhagic transformation. The patient successfully underwent aortic surgery on day 5 and was discharged with a remarkable improvement in his symptoms. Delayed surgery may avoid hemorrhagic transformation in patients with AD-induced IS who have received tPA.
\end{abstract}

Key words: aortic dissection, hemorrhagic transformation, ischemic stroke, recombinant tissue plasminogen activator

(Intern Med 56: 2343-2346, 2017)

(DOI: 10.2169/internalmedicine.8438-16)

\section{Introduction}

Aortic dissection (AD) is a life-threatening emergency in which patients usually present with sudden severe chest and back pain sometimes accompanied by neurological symptoms such as ischemic stroke (IS). Recombinant tissue plasminogen activator (tPA) is approved for the treatment of acute IS within 4.5 hours of onset. The eligibility for tPA treatment according to the current guidelines in the US, Europe, Canada, and Australia (1-4) is based on the criteria adapted from National Institute of Neurological Disorders and Stroke (NINDS) rt-PA Stroke Study (5). In that study, patients who have arterial puncture at a noncompressible site are not indicated to receive this treatment. Previous reports strongly suggest that AD should be considered as a cause of IS before using tPA (6-11). According to the current Japanese guidelines, if a patient with IS is diagnosed with AD, tPA should not be administered (12). However, a precise diagnosis of $\mathrm{AD}$ is sometimes difficult to achieve within the narrow time window of tPA administration because the patients sometimes lack typical medical histories or findings. If tPA is misused in patients with AD-induced IS, emergency aortic surgery may be required (9-11). However, several reports have recommended delaying surgery until the patient's neurologic condition stabilizes because of concerns regarding hemorrhagic transformation (HT) with high-dose heparin for extracorporeal circulation (13-16). We herein report a case involving a patient with $\mathrm{AD}$-induced IS who was treated with tPA and underwent delayed surgical treatment with a remarkable outcome.

\section{Case Report}

A 60-year-old man developed sudden left hemiparesis and visited a nearby hospital 60 minutes after onset. On neurologic examination, he was awake exhibited left hemiparesis and left facial palsy, and had a blood pressure (BP) of $128 / 64 \mathrm{mmHg}$, pulse of $66 \mathrm{bpm}$, body weight of $86 \mathrm{~kg}$, and National Institute of Health Stroke Scale (NIHSS) score of

${ }^{1}$ Departments of Neurology, Dentistry, and Pharmaceutical Sciences, Graduate School of Medicine, Okayama University, Japan and ${ }^{2}$ Department of Neurology, Okayama National Hospital Medical Center, Japan

Received: October 22, 2016; Accepted: December 26, 2016; Advance Publication by J-STAGE: August 10, 2017

Correspondence to Dr. Koji Abe, p2k07119@cc.okayama-u.ac.jp 

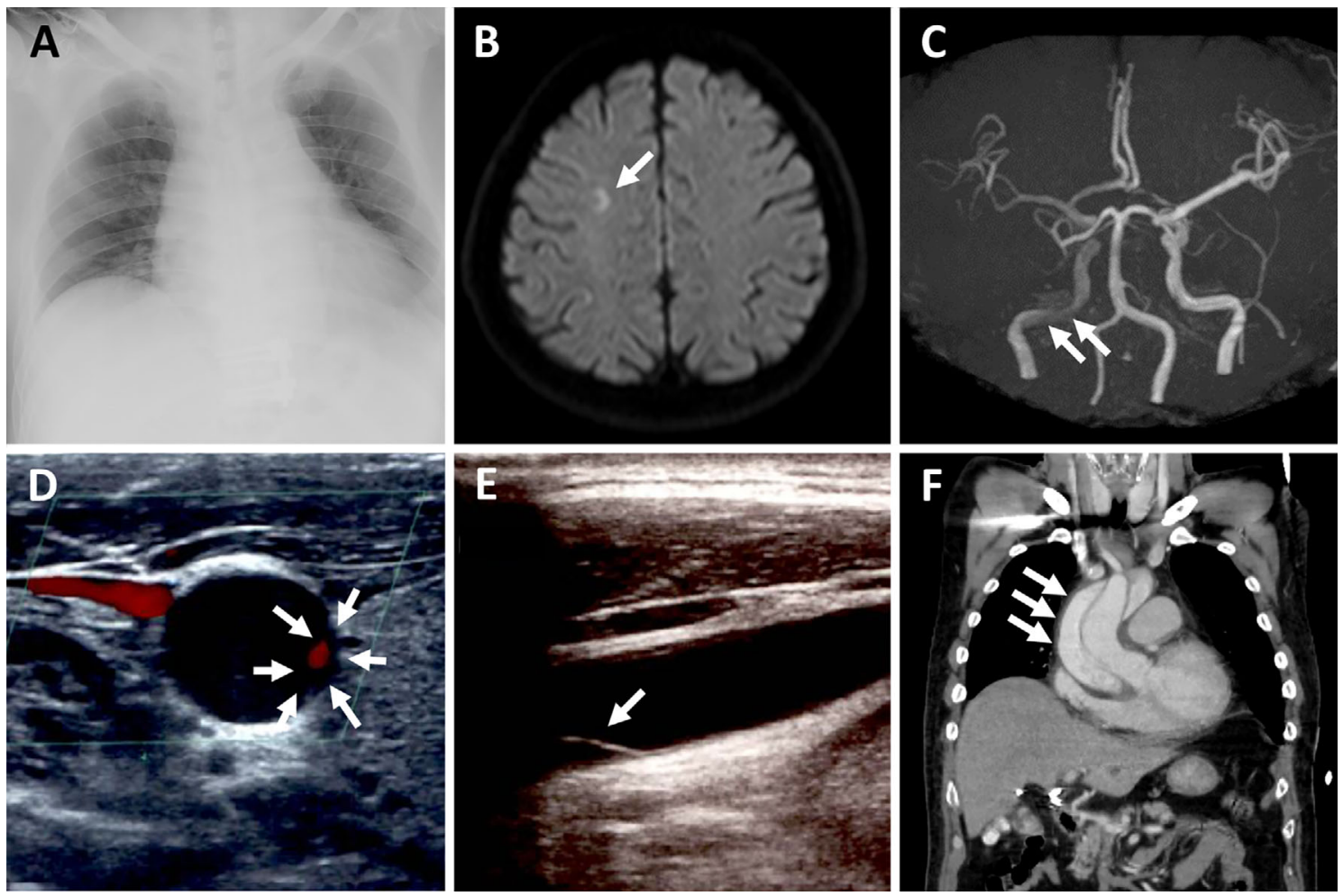

Figure. (A) A supine chest X-ray (anteroposterior view) showed no abnormal findings, including no enlargement of the mediastinum. (B) Brain magnetic resonance imaging revealed a right frontoparietal ischemic lesion (arrow). (C) Brain magnetic resonance angiography demonstrated a low signal in the right internal carotid artery (arrows). (D) Carotid duplex ultrasonography demonstrated 95\% occlusion of the right common carotid artery with a large false lumen and small true lumen (arrows), and a dissecting intima was present in the left internal carotid artery (arrow, E). (F) Chest computed tomography revealed a Stanford type $A$ ascending aortic dissection extending to the aortic arch (arrows).

2. A supine chest X-ray (Figure A) and electrocardiogram were normal, and general serum tests were unremarkable. Brain magnetic resonance imaging revealed a right watershed infarct located on the border of the anterior cerebral artery and middle cerebral artery territories (Figure B, arrow). Brain magnetic resonance angiography demonstrated a low signal in the right internal carotid artery (Figure C, arrows). Based on the diagnosis of IS, infusion of tPA was started at a dose of $0.6 \mathrm{mg} / \mathrm{kg}$ (total, $51.6 \mathrm{mg}$ ) 137 minutes after onset. During the tPA infusion, the patient's BP dropped to 72/ $53 \mathrm{mmHg}$, consciousness declined, and left hemiparesis progressed. Thus, the tPA was stopped after infusing $75 \%$ of the total dose $(38.7 \mathrm{mg})$, and he was emergently transferred to our hospital.

On admission to our hospital, the patient's NIHSS score worsened to 26 with a Glasgow coma scale score of E2V2M5. His BP was $75 / 50 \mathrm{mmHg}$ in his right arm but $140 / 80 \mathrm{mmHg}$ in his left arm. Repeated interviews revealed slight right shoulder pain before his consciousness decline. He was temporally intubated and ventilated. Carotid duplex ultrasonography demonstrated $95 \%$ occlusion of the right common carotid artery with a large false lumen and small true lumen (Figure D, arrows), and a dissecting intima was present in the left internal carotid artery (Figure E, arrow).
Chest computed tomography revealed a Stanford type A ascending AD extending to the aortic arch (Figure F, arrows). Emergency surgery was postponed due to concerns regarding the risk of HT induced by high-dose heparin for extracorporeal circulation. With conventional medical treatment, the patient was removed from the ventilator with a BP of 109/42 mmHg and Glasgow coma scale score of E4V5M6 on admission day 2. He underwent open surgery for reconstruction of the ascending aorta and proximal aortic arch on day 5. After the establishment of full cardiopulmonary bypass with 24,000 units of unfractionated heparin, aortic cross-clamping was achieved and deep hypothermic circulatory arrest was induced. The intimal and medial layers of the false lumen were sealed, and the proximal end of the ascending aorta and proximal aortic arch (between the innominate artery and left common carotid artery) were reconnected by a graft. His BP was maintained at 100-120/50-60 during the surgery. The surgical duration was 9 hours, and he recovered with clear consciousness. Follow-up brain computed tomography on day 10 showed no intracranial hemorrhage. On day 46, he was transferred to a rehabilitation hospital with a remarkable improvement in his NIHSS score from 26 to 1 . At the 3-month follow-up, the patient was living at home and able to carry out all usual activities 
despite slight muscle weakness in the left hand with a modified Rankin scale score of 1.

\section{Discussion}

The eligibility for tPA treatment at $0.9 \mathrm{mg} / \mathrm{kg}$ according to the current guidelines in the US, Europe, Canada, and Australia (1-4) is based on the selection criteria from NINDS rt-PA Stroke Study (5). In the study, patients who have arterial puncture at a noncompressible site are not indicated for this treatment. According to the current Japanese 2012 guidelines, a diagnosis of AD should be excluded before administering tPA at $0.6 \mathrm{mg} / \mathrm{kg}$ (12). The misuse of tPA to treat IS due to $\mathrm{AD}$ may have catastrophic consequences. There are several reports of IS patients receiving tPA with unrecognized AD who took a sudden turn for the worse after tPA, leading to death $(6,7,17)$. However, the treatment of $\mathrm{AD}$-induced IS is challenging in the era of tPA because the diagnosis of $\mathrm{AD}$ is sometimes difficult within the limited time window (up to 4.5 hours) of stroke onset. One-third of patients with $\mathrm{AD}$ who develop neurological symptoms do not report typical pain at onset (18), as was found in the present case at the nearby hospital. Because it is difficult to conduct all diagnostic tests for $\mathrm{AD}$ in every patient who is eligible for tPA, clinical suspicion and physical examination findings are important, especially in patients with a consciousness decline. In the present case, an examination of the bilateral BP at a local hospital may have led to a faster diagnosis, but repeated history-taking, physical examination, and careful conduction of carotid duplex ultrasonography led to a definitive diagnosis after transfer to our hospital.

Previous reports have described eight cases of tPA therapy for AD-induced IS. Three of these patients died without aortic surgery $(6,7,17)$, and the remaining five survived after aortic surgery $(8-11,19)$. Of the five survivors, four underwent emergency surgery on the same day as IS onset (8-11), and one underwent surgery on day 3 because of complications of jugular vein thrombosis and arrhythmia (19). Surgery is the gold standard treatment for AD, but it may be associated with high morbidity and mortality in patients with AD-induced IS who have received tPA. Stroke is not a contraindication for emergent surgery to treat acute type A and B ADs, and the former should be done as soon as possible in all patients except those with profound or evolving neurological deficits (20-24). Because the possibility of HT is a concern when administering high-dose heparin for extracorporeal circulation, several reports have suggested delaying surgery until the patient's neurologic condition stabilizes (13-15). Fukuda et al. (16) reported good results in some patients with brain edema due to AD-induced IS by delayed surgery. In the present case, we delayed performing aortic surgery because of concerns over the risk of HT, and the patient successfully underwent reconstruction of the ascending aorta without any neurological complications.

In conclusion, the present case suggests that $\mathrm{AD}$ should be carefully considered as a cause of IS before administrat- ing tPA. The misuse of tPA to treat IS patients due to AD can lead to a very poor outcome. We delayed the aortic surgery for 5 days until the risk of HT was reduced. The patient successfully underwent the aortic surgery and was discharged with a remarkable improvement in his neurologic manifestations. Delayed surgery could therefore be an alternative choice to avoid HT in patients with AD-induced IS who have received tPA.

The authors state that they have no Conflict of Interest (COI).

\section{Acknowledgement}

This work was supported in part by Grants-in-Aid for Scientific Research (B) 2529320216 and (C) 24591263; a Grant-inAid for Challenging Research 24659651 from the Ministry of Education, Culture, Sports, Science, and Technology; and by Grants-in-Aid from the Research Committees (H. Mizusawa, I. Nakano, M. Nishizawa, H. Sasaki, and M. Aoki) from the Ministry of Health, Labor, and Welfare of Japan.

\section{References}

1. Adams HP Jr, del Zoppo G, Alberts MJ, et al. Guidelines for the early management of adults with ischemic stroke: a guideline from the American Heart Association/American Stroke Association Stroke Council, Clinical Cardiology Council, Cardiovascular Radiology and Intervention Council, and the Atherosclerotic Peripheral Vascular Disease and Quality of Care Outcomes in Research Interdisciplinary Working Groups: the American Academy of Neurology affirms the value of this guideline as an educational tool for neurologists. Stroke 38: 1655-1711, 2007.

2. European Stroke Organization (ESO) Executive Committee; ESO Writing Committee. Guidelines for management of ischaemic stroke and transient ischaemic attack 2008. Cerebrovasc Dis 25: 457-507, 2008.

3. Casaubon LK, Boulanger JM, Blacquiere D, et al. Canadian Stroke Best Practice Recommendations: Hyperacute Stroke Care Guidelines, Update 2015. Int J Stroke 10: 924-940, 2015.

4. Clinical guidelines for stroke management 2010. Stroke Foundation web site [Internet]. [cited 2016 Oct 22]. Available from http $\mathrm{s}$ ://informme.org.au/guidelines/clinical-guidelines-for-stroke-manag ement-2010

5. The National Institute of Neurological Disorders and Stroke rt-PA Stroke Study Group. Tissue plasminogen activator for acute ischemic stroke. N Engl J Med 333: 1581-1587, 1995.

6. Fessler AJ, Alberts MJ. Stroke treatment with tissue plasminogen activator in the setting of aortic dissection. Neurology 54: 1010, 2000.

7. Rodriguez-Luna D, Vilar RM, Peinazo $M$, et al. Intravenous thrombolysis in an elderly patient with acute ischemic stroke masking aortic dissection. J Stroke Cerebrovasc Dis 20: 559-561, 2011.

8. Uchino K, Estrera A, Calleja S, Alexandrov AV, Garami Z. Aortic dissection presenting as an acute ischemic stroke for thrombolysis. J Neuroimaging 15: 281-283, 2005.

9. Chua CH, Lien LM, Lin CH, Hung CR. Emergency surgical intervention in a patient with delayed diagnosis of aortic dissection presenting with acute ischemic stroke and undergoing thrombolytic therapy. J Thoracic Cardiovasc Surg 130: 1222-1224, 2005.

10. Hong KS, Park SY, Whang SI, et al. Intravenous recombinant tissue plasminogen activator thrombolysis in a patient with acute ischemic stroke secondary to aortic dissection. J Clin Neurol 5: 49-52, 2009. 
11. Mendes A, Mendonca T, Sousa A, Moreira G, Carvalho M. Stroke secondary to aortic dissection treated with a thrombolytic: a successful case. Neurol Sci 33: 107-110, 2012.

12. Minematsu K, Toyoda K, Hirano T. Guidelines for the intravenous application of recombinant tissue-type plasminogen activator (alteplase), the second edition, October 2012: a guideline from the Japan Stroke Society. J Stroke Cerebrovasc Dis 22: 571-600, 2013.

13. Piccione W Jr, Hamilton IN, Najafi H. Intentional delayed repair of acute dissection of the ascending aorta complicated by stroke. J Thoracic Cardiovasc Surg 109: 807-808, 1995.

14. Ergin MA, Galla JD, Lansman S, Griepp RB. Acute dissections of the aorta. Current surgical treatment. Surg Clin North Am 65: 721741, 1985.

15. Deeb GM, Williams DM, Bolling SF. Surgical delay for acute type A dissection with malperfusion. Ann Thorac Surg 64: 16691675; discussion 1675-1677, 1997.

16. Fukuda I, Imazuru T. Intentional delay of surgery for acute type A dissection with stroke. J Thorac Cardiovasc Surg 126: 290-291, 2003.

17. Takeuchi S, Takasato Y, Masaoka H, Otani N. Administration of recombinant tissue plasminogen activator to a case of cerebral infarction in the setting of painless aortic dissection. Neurol India 57: 808-809, 2009.

18. Gaul C, Dietrich W, Erbguth FJ. Neurological symptoms in aortic dissection: a challenge for neurologists. Cerebrovasc Dis 26: 1-8,
2008.

19. Noel M, Short J, Farooq MU. Thrombolytic therapy in a patient with acute ischemic stroke caused by aortic dissection. Clin Neurol Neurosurg 112: 695-696, 2010.

20. Estrera AL, Garami Z, Miller CC, et al. Acute type A aortic dissection complicated by stroke: can immediate repair be performed safely? J Thorac Cardiovasc Surg 132: 1404-1408, 2006.

21. Nakamura Y, Tagusari O, Ichikawa Y, Morita A. Impact of immediate aortic repair on early and midterm neurologic status in patients with acute type a aortic dissection complicated by cerebral malperfusion. Ann Thorac Surg 92: 336-338, 2011.

22. Tsukube T, Hayashi T, Kawahira T, et al. Neurological outcomes after immediate aortic repair for acute type A aortic dissection complicated by coma. Circulation 124: S163-S167, 2011.

23. Morimoto N, Okada K, Okita Y. Lack of neurologic improvement after aortic repair for acute type A aortic dissection complicated by cerebral malperfusion: predictors and association with survival. J Thorac Cardiovasc Surg 142: 1540-1544, 2011.

24. Orihashi K. Acute type a aortic dissection: for further improvement of outcomes. Ann Vasc Dis 5: 310-320, 2012.

The Internal Medicine is an Open Access article distributed under the Creative Commons Attribution-NonCommercial-NoDerivatives 4.0 International License. To view the details of this license, please visit (https://creativecommons.org/licenses/ by-nc-nd/4.0/).

(C) 2017 The Japanese Society of Internal Medicine Intern Med 56: 2343-2346, 2017 